\title{
DERECHO ROMANO: COMPARACIÓN CON EL DERECHO ACTUAL EN LOS MÉTODOS POSITIVOS PARA GESTIONAR CONFLICTOS
}

\author{
Gema Vallejo Pérez \\ Universidad de León (España) \\ gvalp@unileon.es \\ María Consuelo Morán Astorga \\ Universidad de León (España)
}

Fecha de Recepción: 16 Marzo 2019

Fecha de Admisión: 30 Abril 2019

\section{RESUMEN}

Procedimientos alternativos a la vía judicial existieron en el Derecho Romano para resolver controversias; muchas de ellas, surgidas en el entorno familiar y en ámbito de los negocios. En la actualidad, a través de la Mediación, de la Negociación y del Arbitraje (Alternative Dispute Resolution ADR) se pueden obtener acuerdos más favorables para los intereses de ambas partes. Además, estos métodos ADR protegen la privacidad y evitan disgustos que perjudican la salud y destruyen las buenas relaciones futuras entre las partes. El objetivo de este trabajo fue comparar las estrategias alternativas a la Jurisdicción existentes en la Época Romana con los métodos ADR empleados en la actualidad. Este estudio meta-analítico consistió en la revisión de la normativa y de documentos bibliográficos romanos y compararlos con las fuentes del Derecho actual. Los resultados informan que, tanto en el Derecho Romano como en el Derecho Actual, las estrategias ADR alternativas a la vía judicial presentan importantes ventajas. Se pueden destacar: la preservación de la privacidad, el buen acogimiento de la resolución por las partes, una gestión más positiva de las emociones, y una conservación de las buenas relaciones entre las partes. Tanto en la época romana como en nuestros tiempos, estos aspectos tuvieron especial relevancia en el ámbito de la familia y también en los negocios privados.

Palabras clave: derecho romano; ADR; gestión del conflicto; mediación; negociación; arbitraje

\section{ABSTRACT}

Roman law: comparison with current law in positive methods to manage conflicts. Alternative procedures to the judicial way existed in the Roman Law to solve controversies; many of them emerged in the family environment and in the business world. At present, through Mediation, Negotiation and Arbitration (Alternative Dispute Resolution - ADR), more favorable agreements can 
be obtained for the interests of both parties. In addition, these ADR methods protect privacy and avoid distress that damage the health and destroys the future relationships between the parties. The objective of this work was to compare the alternative strategies existing in the Roman Jurisdiction with the ADR methods used at present. This meta-analytical study consisted in the bibliographic revision of roman documents to compare them with the sources of current Law. The results inform that, in both, Roman Law and Current Law, alternative strategies like ADR, offers important advantages to the judicial way. They can be highlighted: the preservation of privacy, the good reception of the resolution by the parties, a more positive management of emotions, and a preservation of good relations between the parties. Both in Roman times and in our times, these aspects have special relevance in the field of the family and also in private businesses.

Keywords: roman law; ADR; conflict management; mediation; negotiation; arbitration

\section{EL DERECHO POSITIVO}

Peterson y Seligman (2004) clasifican y describen las fortalezas y virtudes que permiten el crecimiento humano. El esquema general se basa en seis virtudes principales que casi todas las culturas en el mundo aprueban, como son: sabiduría, valor-coraje, humanidad, justicia, moderación y trascendencia. Bajo cada virtud hay fortalezas particulares para practicarlas.

La cuarta virtud es la JUSTICIA definida como las fortalezas cívicas que son base de la vida sana de la comunidad. Las fortalezas que la acompañan son:

Ser justo: Tratar a toda la gente con imparcialidad y justicia. No dejar que los sentimientos personales influyan en las decisiones sobre otros, dar a todo el mundo las mismas oportunidades.

Liderazgo: Organizar actividades para el grupo y llevarlas a buen término. Animar al grupo de pertenencia a hacer cosas, reforzar las buenas relaciones.

Trabajo en equipo: Trabajando bien como miembro de un equipo, ser fiel al grupo y sentirse parte de él.

También está dentro de los planes de la psicología positiva el expandir sus principios a otros campos como la economía, la sociología, la política, el derecho, la filosofía, y conformar un campo que podría denominarse como Ciencias Sociales Positivas.

Es en este sentido donde se enmarca nuestro trabajo, aunando dos disciplinas que en su compromiso tienen el de resolver conflictos, problemas y cuestiones que, si no se solucionan positivamente en el nivel personal, familiar o de grupo social pueden dar lugar a buscar su gestión por la vía judicial ${ }^{1}$. En cualquier caso, son problemas humanos que han de ser resueltos para que las personas sigan con su funcionamiento normal y puedan desarrollarse y ser felices.

Es la mediación un método de resolución de conflictos que se enmarca dentro de los pasos previos 0 alternativos a la vía judicial.

Ya en la antigua Roma hubo formas de resolver conflictos de manera alternativa. Entre ellas existió el iudicium domesticum ${ }^{2}$ o consilium domesticum que era un órgano consultivo, un instrumento al servicio de la familia, pero de utilización interna, que carecía de las facultades de un tribunal. La denominación de consilium domesticum ayuda a comprender que el significado de consejo suponía la asunción de un órgano dentro de la unidad familiar, que apoyaba al paterfamilias. En determinadas ocasiones, para aconsejarlo a la hora de tomar una decisión con respecto a uno de los sujetos sometidos a la potestad paterna, que se había alejado del código de conducta requerido como aceptable o positivo dentro de la familia.

El consilium domesticum era un consejo de familiares o amigos cercanos, que servía para asesorar al paterfamilias ${ }^{3}$ en las decisiones más importantes que afectaban al seno de la familia; también sirvía para refrendar los actos potestativos del cabeza de familia. La historia del derecho roma- 
no da cuenta, al menos de la presencia de un órgano que mitigaba el poder absoluto que conocíamos con respecto al paterfamilias, refrendado por el ius romanorum.

La realidad del consilium domesticum añade una nota de modernidad al derecho relativo a la familia que viene de la antigua Roma 4 . La relación del consilium domesticum con el derecho actual y con la mediación familiar existe precisamente por causa de ese legítimo deseo de preservar a la familia de la publicidad negativa externa. En otras palabras, la mediación se produce precisamente en casos en los que se desea evitar un juicio para preservar la intimidad de los que acceden a la mediación, que pretenden así evitar el escarnio público 0, mejor dicho, la exposición externa de sus conflictos familiares.

El consilium domesticum, como órgano interno para resolver conflictos en la familia romana, lo podemos tomar como un antecedente de la moderna mediación, como un modo alternativo de resolución de controversias, que ayuda a la desaparición de graves problemas en el seno familiar.

En España, existe, como referente a este consejo familiar la 'Junta de parientes' 5 en el derecho aragonés, que es una especie de consejo familiar que decide ante asuntos delicados concernientes a la familia, como sucedía en la antigua Roma. Los que se someten a esta Junta de parientes deben aceptar el resultado adoptado por la misma, a diferencia del consilium domesticum en el que el protagonismo lo tenía el padre. Ambos muestran la diferencia con la mediación familiar ya que aquí la decisión la toman las partes implicadas.

\section{LA MEDIACIÓN}

La mediación, a pesar de que parece un instrumento jurídico moderno y de reciente creación, hunde sus raíces en el Derecho Romano. El Derecho Romano puede considerarse como una verdadera escuela de libertad creativa jurídica, y es actualmente imprescindible conocerla para conseguir la universalidad del sentido jurídico, en donde ya existían procedimientos alternativos para resolver conflictos, muchos de ellos provenientes del entorno familiar6.

La mediación es un proceso en el que las partes en conflicto, ayudadas por un mediador, llegan a una solución razonablemente satisfactoria para ambas 7 .

La mediación procura mejores acuerdos que los que se pueden obtener por vía judicial, al igual que con el consilium domesticum, y además procura la salvaguarda de la intimidad de la propia familia.

El acuerdo de mediación ${ }^{8}$, sin ser mejor que el logrado por la vía judicial, es más beneficioso porque las dos partes quedan en buena medida más satisfechas mientras que en por la vía judicial una de las partes sale ganando a expensas de que la otra pierda.

Aunque en ocasiones, la mediación, como herramienta equitativa, dejará menos satisfechas a ambas partes en alguna medida, pero con una sentencia judicial es evidente que alguien sería más perjudicado.

También la mediación familiar moderna tiene sus raíces en el ius romanorum, cuyo valor tan unido a la interpretación creativa de la jurisprudencia dio lugar a la resolución de diversos conflictos sin la intervención judicial.

Y la mediación familiar persigue el mismo objetivo, en el sentido de procurar la mejor solución en un conflicto familiar, pero eso sí, dejando el protagonismo absoluto a las partes, que son las que deben decidir en un plano de igualdad cómo pueden terminar con su conflicto, eliminando las dificultades y procurando un acuerdo equitativo que los aleje de la judicialización de sus diferencias.

\section{BENEFICIOS DE LA MEDIACIÓN}

La principal ventaja de la mediación es que las partes diseñan un procedimiento de comunicación y de resolución de sus discrepancias a la medida de sus necesidades, intereses y pretensiones, 
ayudadas por un mediador, sin tener que acudir a la vía judicial. Esto contribuye a dejar fuera del ámbito de los tribunales de justicia aquellos asuntos que muchas veces no precisan una judicialización. Por ello, la mediación despliega su máxima utilidad cuando se aplica apropiadamente a la resolución de determinados conflictos, actuando como un método no excluyente de la jurisdicción, sino complementario, menos costoso y rápido que las actuaciones ante los tribunales.

Es la mediación un método confidencial ${ }^{9}$ en el cual se propicia un ámbito de intimidad en el que las partes se desahogan y pueden expresar sus sentimientos, asistiendo el tercero, mediador, como un catalizador para que los involucrados en la controversia hallen la solución más idónea a sus problemas. Este tercero en el procedimiento de mediación no resuelve el conflicto, su labor consiste en ayudar a las partes a resolver sus diferencias y a encauzar sus relaciones para el futuro.

El mediador, manteniéndose siempre neutro e imparcial ${ }^{10}$ ante las partes, no impone una solución al problema, son las partes quienes deben tomar sus propias decisiones y alcanzar por sí mismas un acuerdo que les satisfaga a ambas. Estas particularidades ponen de relieve que, respecto a la vía judicial, la mediación tiene la ventaja de que las partes pueden modificar sus pretensiones. Esto es, la petición en la mediación puede variar y adaptarse a los intereses cambiantes de las partes, a diferencia de la vía judicial que debido al principio de congruencia debe mantenerse inalterable durante todo el proceso y el juez debe pronunciarse solo sobre la inamovible petición.

Por otro lado, si las partes llegan a un acuerdo total o parcial, éste, por fuerza, debe satisfacer a ambas, siendo de más fácil cumplimiento por haber sido consensuado en un procedimiento autocompositivo, en el que no hay vencedores ni vencidos; y aún en el caso de que el procedimiento de mediación fracase, siempre cabe acudir a la jurisdicción ${ }^{11}$.

En la mediación, el mediador actúa como un tercero que intenta acercar y guiar la comunicación entre las partes con el fin de que lleguen a un acuerdo eficaz. Dicho acuerdo se materializará en un documento escrito que tiene fuerza y que podrá ser elevado a escritura pública, pero que no tendrá, lógicamente, valor de cosa juzgada.

La mediación es más acertada para aquellos conflictos en los que las partes valoren la confidencialidad 0 donde tengan la necesidad de resolver el conflicto de forma rápida y a la vez la solución tomada mantenga la continuidad de la relación entre las partes.

La mediación es recomendable para aquellos conflictos donde las partes conozcan como nadie sus verdaderos intereses y sus pretensiones y aquello en lo que pueden ceder para conducir a un acuerdo satisfactorio para ambas. De la misma forma, es favorable por la necesaria aplicación del principio de flexibilidad y del respeto a la autonomía para la resolución de este tipo de conflictos. El acuerdo alcanzado podrá tener la consideración de título ejecutivo, si las partes así lo quieren, mediante elevación a escritura pública (si se trata de una mediación extrajudicial) o mediante auto del juez (si se trata de una mediación intrajudicial).

En cuanto a los inconvenientes en la aplicación de la mediación, debemos resaltar que si se trata de una Mediación Extrajudicial y las partes no lo elevan a escritura pública, tendrá, al igual que la negociación, valor contractual cuyo eventual incumplimiento deberá solventarse mediante la interposición de la correspondiente demanda ante el juzgado competente para obtener el cumplimiento de lo pactado.

La mediación no es el único método de positivo de resolución de problemas al que las partes pueden acudir para solucionar sus conflictos, existen otros métodos alternativos a la vía jurídica como son la negociación, la conciliación y el arbitraje. Su ámbito de aplicación son las materias de libre disposición, junto con el principio de autonomía de la voluntad entre ellos. 


\section{OTROS MEDIOS ALTERNATIVOS A LA VÍA JUDICIAL}

La conciliación ${ }^{12}$ es también un proceso alternativo a la vía judicial en el que hay un tercero que intenta acercar las posturas de las partes. El conciliador propone fórmulas de solución no obligatorias que ayudan a las partes a llegar a un acuerdo, el mediador no tiene facultad para proponer opciones de solución al conflicto, solo facilita que éstas lleguen por sí mismas a un acuerdo.

La conciliación se inserta dentro de un acto jurídico, por medio del cual las partes se someten -antes de un proceso o en el transcurso de él- a un trámite conciliatorio, a celebrar ante un letrado de la administración de justicia de los Juzgados de Primera Instancia 0 de lo Mercantil 0 ante el Juzgado de Paz, a fin de que se llegue a un acuerdo que tendrá carácter de cosa juzgada y valor ejecutivo. Respecto a la conciliación en el ámbito laboral, el hecho de que sea obligatoria tal vez ha desvirtuado un tanto su eficacia llegando a ser en muchas ocasiones un mero trámite procesal más, que da acceso a la vía judicial para acabar resolviéndose el conflicto mediante sentencia.

La negociación ${ }^{13}$, otro proceso alternativo a la vía judicial, en el que las partes en conflicto también intentan alcanzar un acuerdo que lo solvente, pero en este caso, el procedimiento se lleva a cabo sin la presencia de un tercero, aunque puede realizarse a través de representantes con autorización para negociar y llegar a pactar un acuerdo, que después podrá ser aprobado o rechazado por éstas.

La negociación, al igual que los anteriores, puede iniciarse antes o durante el proceso judicial, suspendiendo el proceso judicial. Si la negociación tiene éxito y se llega a un acuerdo, se podrá firmar el acuerdo que equivale a un contrato, sometido a las normas que rigen en el Código civil, y en caso de incumplimiento se podrá recurrir a la jurisdicción.

Por lo que respecta al arbitraje ${ }^{14}$, las partes en disputa deciden, de forma voluntaria, que ésta sea dirimida definitivamente por un tercero, llamado árbitro, con la emisión de una decisión vinculante escrita llamada 'laudo'. El laudo tiene valor de cosa juzgada y, se podrá, por vía ejecutiva, exigir su cumplimiento judicial. La ventaja fundamental del arbitraje frente a un procedimiento judicial es la rapidez para solucionar un conflicto mediante un laudo que tiene las mismas garantías que una sentencia judicial. El arbitraje no es un procedimiento tan flexible como el de un acuerdo de mediación, y en el que inevitablemente, por decidir la cuestión un tercero, las partes perderán todo control en la solución adoptada en el laudo.

Estos métodos agilizan la sobrecarga de los juzgados no solo porque no se llegaría a sustanciar asuntos que no precisan judicialización, sino también porque se simplificaría el trabajo de los juzgados al verse reducido el número de incumplimientos de sentencia evitando ejecuciones y disminuyendo el número de procesos contenciosos. A la vez que constituyen unos métodos más ágiles para solucionar los conflictos.

Con todo, esos métodos anteriormente expuestos, suponen un gasto para las partes y, en algunos casos como en el arbitraje puede ser costoso, a lo que cabe añadir los honorarios de los abogados. Mientras, algunos sectores doctrinales están considerando que la vía judicial sólo quedará para quien no pudiese costearse estos métodos alternativos de justicia "privada".

Otro sector doctrinal entiende el problema de la manera contraria, ya que por ser el proceso judicial a la larga más caro, salvo que se litigue con el beneficio de justicia gratuita, los métodos alternativos quedarían como una justicia de segunda destinada a quienes carecen de medios suficientes para acceder a la justicia "pública".

Los métodos alternativos ${ }^{15}$ a la vía judicial contribuyen a presentar una variedad de servicios según las circunstancias y tipo de conflicto, pues estos métodos no son adecuados para todos los conflictos. Una de las labores de los letrados seria aconsejar a sus patrocinados sobre la forma más adecuada de resolver su conflicto. Esto requiere una mayor implicación de estos profesionales con 


\section{DERECHO ROMANO: COMPARACIÓN CON EL DERECHO ACTUAL EN LOS MÉTODOS POSITIVOS PARA GESTIONAR CONFLICTOS}

la mediación y parece que a ello apunta el Manifiesto presentado por el Consejo de la Abogacía en enero de 2018.

\section{PRESENTE Y FUTURO DE LA MEDIACIÓN}

La Mediación Intrajudicial está teniendo cierto auge gracias a los proyectos piloto y a los convenios del Consejo General del Poder Judicial (CGPJ), ya que los Jueces y Letrados de la Administración de Justicia han accedido a implantar determinados proyectos de mediación en sus juzgados.

La Guía para la Práctica de la Mediación Intrajudiacial del CGPJ16 recomienda como "obligatoria" la asistencia a la sesión informativa en los procedimientos judiciales, lo que ha contribuido a la expansión de la Mediación Intrajudicial. También, se ha de mencionar que los notarios ya incluyen en sus documentos cláusulas de acceso a la mediación para la resolución de posibles discrepancias.

Muchos sectores empiezan a apostar por la mediación: aseguradoras, centros educativos, centros sanitarios, centros penitenciarios..., en la actualidad. La Mediación Extrajudicial no está tan implantada como la Intrajudicial, pese a las ventajas que pueda presentar esta para resolver conflictos.

La solución pasa por establecer lo que se puede llamar "voluntariedad mitigada"17 u "obligatoriedad mitigada" que consiste en exigir a las partes que acudan a una sesión informativa sobre la mediación, esto debe ser un requisito previo de acceso a los Tribunales.

Otro factor que contribuye a la expansión de mediación es garantizar la calidad de la misma mediante la cualificación de profesionales para ejercer las funciones de mediador. También, se ha propuesto que acudir al proceso de mediación reporte beneficios fiscales, al igual que en otros países de la Unión Europea.

Hoy día se puede acudir a la mediación para los asuntos civiles, mercantiles y en materia de derecho de familia.

También debemos señalar que el ámbito material de la mediación sigue avanzando con la Justicia restaurativa, centrada en la reparación de la víctima, que tan buen resultado ha dado con los menores. La Ley 1/2015, de 12 de febrero, del Servicio Regional de Mediación Social y Familiar de Castilla-La Mancha, tras su reforma, ha añadido un procedimiento específico de mediación para favorecer la búsqueda de acuerdos entre la víctima y el infractor y para la reparación de la responsabilidad penal en el caso de que el delito haya sido cometido por un menor.

A pesar de las ventajas de la mediación, en el ámbito penal tiene un tope en los asuntos de violencia de género. No solo porque la Ley Orgánica 1/2004, de 28 de diciembre, de Medidas de Protección Integral contra la Violencia de Género expresamente lo prohíba (Art. 44.5), sino porque su aplicación a los delitos en este ámbito no resultaría conveniente porque existe, desde el principio, una desigualdad evidente entre ambas partes 18 .

En el caso de que se intentara su aplicación en casos de violencia de género, solo se utilizará en aquellos en los que el equilibrio de las partes esté garantizado y se trate de delitos muy leves. Algunos profesionales creemos que, en los casos de violencia de género, no debe aplicarse nunca.

El futuro de la mediación tiene un reto: unificar criterios en cuanto a las formas de acceso a la profesión de mediador en la Unión Europea ${ }^{19}$. Cada país comunitario exige unos requisitos y esto es algo que debe unificarse. Cada estado miembro ha establecido una formación inicial y continua con diversas características. Unos consideran una formación de 400 horas, otros de 200 y otros de menos de 100. Y, junto a una formación general se tiende a exigir y una formación especializada. Otro aspecto sustancial que varía de país a país es el de si la mediación debe ser de carácter voluntario u obligatorio. 
En España convendría revisar el Estatuto del Mediador y la formación del mediador, porque el Ministerio de Justicia no exige ninguna titulación, ni formación específica para ser mediador. Esto es claramente insuficiente y va contra la Directiva Europea sobre ciertos aspectos de la mediación en asuntos civiles y mercantiles.

Los mediadores no podrán proporcionar un servicio de calidad si no se pone énfasis en su adecuada preparación profesional. Es necesario dar un mayor protagonismo a las Administraciones Públicas en la supervisión y control de la titulación adecuada y la formación continua de los mediadores con el fin de minimizar la indefinición de las personas que pueden ser mediadoras.

Siguiendo con la situación en nuestro país, en relación con la mediación, debemos señalar que, en España, uno de los principales problemas a los nos enfrentamos es la inexistencia de una cultura de mediación, no a su regulación, ya que lo está por las Comunidades Autónomas.

También debemos indicar que es necesario implantar la mediación como servicio público, con gratuidad aparejada, en los casos de personas con rentas bajas. En la Ley de Mediación en Asuntos Civiles y Mercantiles 20 no se contempla la mediación gratuita. La Ley debería contemplar servicios gratuitos y universales de mediación, dependientes de la administración, como lo han hecho las leyes de Mediación Familiar y de Mediación de todas las Comunidades Autónomas.

Además, se deberá valorar la conveniencia de generalizar el turno de oficio de mediación que ya contemplan algunas leyes autonómicas de Mediación Familiar, así como los servicios de mediación que ya ofrecen algunos colegios profesionales y ante los cuales los ciudadanos en donde no existen, podrían alegar agravio comparativo.

De no existir servicios gratuitos de mediación ¿qué sucederá en los casos en que las partes acuden y están de acuerdo con iniciar el proceso de Mediación, pero no pueden pagarlo? ¿Qué sucederá si una de las partes puede pagar y la otra no? 0 ¿qué sucederá en los casos en que el juzgador sugiere una Mediación que es acogida por las partes, pero las partes tienen abogados de oficio, pero no hay un servicio de Mediación gratuito?

El fin de la mediación no es el de desatascar los juzgados, si no el entender los conflictos de una manera diferente. La mediación fomenta la cultura de la paz, ayudando a las personas a responsabilizarse de sus actos y devolviendo al ciudadano su poder de decisión.

Estos aspectos adquieren especial relevancia en la Mediación Familiar, por ser un ámbito en el que hay intereses con los que se debe ser especialmente vigilante: menores, personas mayores y personas con discapacidad; además la mediación vela sobre una materia tan sensible y tan compleja como es la relación familiar.

Por esta razón, es en el ámbito familiar donde los resultados de la aplicar la mediación han mostrado la más alta satisfacción para las partes. Y esto aplicado a conflictos tan complejos como la sustracción internacional de menores u otros. La concreción de conceptos jurídicos indeterminados -como el interés superior del menor- prueban la amplia potencia de la Mediación Familiar a la hora de clarificar aspectos generales del Derecho.

La sólida base que ofrece un Derecho de la condición de alta técnica jurídica como es el Derecho Romano unido a la potencialidad actual que presenta la Mediación Familiar en cuanto instrumento de gestión de conflictos familiares, permite llegar donde algunas veces la generalidad propia de la norma no consigue. Esto nos permite entender que la Mediación tiene ante sí un largo recorrido, en el que deberá perfeccionarse y superar los obstáculos y desafíos propios de una institución, por un lado, tan "antigua" y, por otro, tan "joven" en nuestro Sistema Jurídico. 


\section{REFERENCIAS BIBLIOGRÁFICAS}

BELLOSO MARTín, N., La Mediación Familiar: algunas experiencias en el Derecho Comparado Internacional en Estudios sobre Mediación: La Ley de Mediación Familiar de Castilla y León. AA.VV., BELLOSO MARTín, N. Coordinadora. Valladolid, Primera Edición, Ed. Consejería de Familia e Igualdad de Oportunidades Junta de Castilla y León, 2006.

BRAVO BOSCH, M.J., El mito de Lucrecia y la familia romana, en MULIER. Algunas Historias e Instituciones de Derecho Romano, Madrid, Ed. Dykinson, 2013.

CONSEJO GENERAL DEL PODER JUDICIAL, Guía para la práctica de la mediación intrajudicial, noviembre de 2016. Disponible en: http://www.poderjudicial.es/cgpj/es/Temas/Mediacion/Guiapara-la-practica-de-la-Mediacion-Intrajudicial/.

CONSEJO GENERAL DE LA ABOGACÍA. Abogados de Badajoz 2014, Conclusiones I/ encuentro de abogados y abogadas de violencia de genero, (en línea). Disponible en: http://www.abogacia.es/wp-content/uploads/2015/03/CONCLUSIONES-VG-BADAJOZ.pdf.

FERNÁNDEZ DE BUJÁN, A., Discurso leído el día 20 de enero de 2014, en el acto de su recepción como Académico de número, en la Real Academia de Jurisprudencia y Legislación el día 20 de enero de 2014, Ed. Dykinson, 2014, Madrid.

FERNÁNDEZ DE BUJÁN, A., El filiusfamilias independiente en Roma y en el Derecho español, Madrid, Universidad Autónoma de Madrid, 1984.

GARCÍA VILLALUENGA, L., Mediación en conflictos familiares. Una construcción desde el Derecho de familia, Madrid, Universidad Complutense de Madrid, 2006.

GARCÍA VILLALUENGA, L., VAZQUEZ DE CASTRO, E., La Mediación a debate en Europa ¿Hacia una voluntariedad mitigada? en Anuario de Mediación y solución de conflictos 2015. AA.VV., GARCÍA VILLALUENGA L., VÁZQUEZ DE CASTRO E., Directores, Madrid, Ed. Reus, 2016.

IZAGUIRRE ARTAZA, J., El Arbitraje y la Mediación en Estados Unidos (informe en línea). Ed. ICEX España Exportación e Inversiones, 2014. Disponible en: file:///C:/Users/Gemma/Downloads/4768017.pdf.

Ley $5 / 2012$, de 6 de julio, de mediación en asuntos civiles y mercantiles

MARTIN MingARRO, L., La Mediación Civil y Mercantil en la nueva Ley 5/2012, de 6 de julio en Revista Jurídica de Castilla León, 29, 2013.

MUNNÉ CATARINA, F., Código ético. Modelo de conducta, imparcialidad, neutralidad y deber de confidencialidad en La Mediación. Resolución pacífica de conflictos. Régimen jurídico y eficacia procesal. MUNNE CATARINA, F., VIDAL TEIXIDÓ, A., Autores. Madrid, La Ley, 2013.

ORTUÑO MUÑOZ, P., Introducción: El impulso a la mediación en la experiencia de los PNPM en Mediación es justicia. El impacto de la Ley 5/2012, de Mediación Civil y Mercantil, AA.VV. LAUROBA LACASA, Ma‥ E., ORTUÑO MUÑOZ, P., Coordinadores, Barcelona, Ed. Huygens, 2014.

SAN CRISTÓBAL REALES, S., Sistemas alternativos de resolución de conflictos: negociación, conciliación, mediación, arbitraje, en el ámbito civil y mercantil en Anuario Jurídico y Económico Escurialense, XLVI, 2013.

TAMAYO HAYA, S., El acuerdo de mediación y la formalización del título ejecutivo, en Practicum mediación 2014. VÁZQUEZ DE CASTRO, E., Director, Navarra, Editorial Aranzadi, 2013.

UNIÓN EUROPEA, COMISIÓN DE LAS COMUNIDADES EUROPEAS, Libro Verde sobre las modalidades alternativas de solución de conflictos en el ámbito del Derecho Civil y Mercantil, Bruselas, Unión Europea. Comisión de las Comunidades Europeas, 2002, p. 2. Disponible en: http://www.poderjudicial.es/cgpj/es/Temas/Mediacion/Normativa-y-jurisprudencia/Normativaeuropea/Libro-Verde-sobre-las-modalidades-alternativas-de-solucion-de-conflictos-en-el-ambito-del-derecho-civil-y-mercantil 
VALLEJO PÉREZ, G., Arbitraje y fiscalidad en el Derecho Romano en Revista General de Derecho Romano, IUSTEL, 24, 2015.

VIÑAS, A., Instituciones políticas y sociales de Roma: Monarquía y República, Madrid, Editorial Dykinson, 2007.

\section{NOTAS}

1 FERNÁNDEZ DE BUJÁN, A., Discurso leído el día 20 de enero de 2014, en el acto de su recepción como Académico de número, en la Real Academia de Jurisprudencia y Legislación el día 20 de enero de 2014, Ed. Dykinson, 2014, Madrid.

2 BRAVO BOSCH, M.J., El mito de Lucrecia y la familia romana, en MULIER. Algunas Historias e Instituciones de Derecho Romano, Madrid, Ed. Dykinson, 2013, pp. 34-35.

3 FERNÁNDEZ DE BUJÁN, A., El filiusfamilias independiente en Roma y en el Derecho español, Madrid, Universidad Autónoma de Madrid, 1984 , p. 22.

4 VIÑAS, A., Instituciones políticas y sociales de Roma: Monarquía y República, Madrid, Editorial Dykinson, 2007, p. 81: "El séptimo y último rey de Roma fue Tarquinio el Soberbio. El relato que ofrece Tito Livio lo presenta como hijo o nieto del antiguo rey Tarquinio. Se convierte en yerno de Servio Tulio al contraer matrimonio con una de sus hijas. Secundando la iniciativa de su ambiciosa mujer, logra que el rey sea ejecutado después de arremeter violentamente contra él en una tumultuosa reunión senatorial... Inicia su reinado con tan malas artes que en adelante será conocido por el calificativo de soberbio".

6 García VILlaLUENGA, L., Mediación en conflictos familiares. Una construcción desde el Derecho de familia, Madrid, Universidad Complutense de Madrid, 2006, pp. 176-178.

7 ORTUÑO MUÑOZ, P., Introducción: El impulso a la mediación en la experiencia de los PNPM en Mediación es justicia. El impacto de la Ley 5/2012, de Mediación Civil y Mercantil, AA.VV. LAUROBA LACASA, Ma‥ E., ORTUÑO MUÑOZ, P., Coordinadores, Barcelona, Ed. Huygens, 2014, p. 125.

8 MARTIN MInGARRO, L., La Mediación Civil y Mercantil en la nueva Ley 5/2012, de 6 de julio en Revista Jurídica de Castilla León, 29, 2013, p.7-8

9 IZAGUIRRE ARTAZA, J., El Arbitraje y la Mediación en Estados Unidos (informe en línea). Ed. ICEX España Exportación e Inversiones, 2014. Disponible en: file:///C:/Users/Gemma/Downloads/4768017.pdf. La confidencialidad es el objeto esencial de la UMA, ya que la plena sinceridad de las partes y del mediador será la vía que posibilite que la mediación llegue a buen puerto. "El núcleo central de la UMA radica en el hecho de que la comunicación en la mediación es y está sujeta a confidencialidad y no puede ser objeto ni de investigación ni de utilización como prueba o evidencia en cualquier otro procedimiento que no sea el mediador".

10 MUNNÉ CATARINA, F., Código ético. Modelo de conducta, imparcialidad, neutralidad y deber de confidencialidad en La Mediación. Resolución pacífica de conflictos. Régimen jurídico y eficacia procesal. MUNNE CATARINA, F., VIDAL TEIXIDÓ, A., Autores. Madrid, La Ley, 2013, pp. 86-88.

11 TAMAYO HAYA, S., El acuerdo de mediación y la formalización del título ejecutivo, en Practicum mediación 2014. VÁZQUEZ DE CASTRO, E., Director, Navarra, Editorial Aranzadi, 2013, pp. 192194.

12 BELLOSO MARTín, N., La Mediación Familiar: algunas experiencias en el Derecho Comparado 
Internacional en Estudios sobre Mediación: La Ley de Mediación Familiar de Castilla y León. AA.VV., BELLOSO MARTín, N. Coordinadora. Valladolid, Primera Edición, Ed. Consejería de Familia e Igualdad de Oportunidades Junta de Castilla y León, 2006, pp. 85-86.

13 SAN CRISTÓBAL REALES, S., Sistemas alternativos de resolución de conflictos: negociación, conciliación, mediación, arbitraje, en el ámbito civil y mercantil en Anuario Jurídico y Económico Escurialense, XLVI, 2013, p. 41.

14 VALLEJO PÉREZ, G., Arbitraje y fiscalidad en el Derecho Romano en Revista General de Derecho Romano, IUSTEL, 24, 2015, pp. 24 y ss.

15 ORTUÑO MUÑOZ, P., Introducción: el impulso a la mediación en la experiencia de los PNPM en Mediación es Justicia. El impacto de la Ley 5/2012, de Mediación Civil y Mercantil, AA. VV., LAUROBA LACASA, Mํ.., E., ORTUÑO MUÑOZ, P., Coordinadores, Barcelona, Ed. Huygens, 2014, pp. 8-10.

16 España. Consejo General del Poder Judicial, Guía para la práctica de la mediación intrajudicial, noviembre de 2016. Disponible en: http://www.poderjudicial.es/cgpj/es/Temas/Mediacion/Guiapara-la-practica-de-la-Mediacion-Intrajudicial/. "a. El Magistrado considera apropiada la mediación para el caso y recomienda a las partes que se pongan en contacto con el Servicio de Mediación, para que sean informados. b. Se puede facilitar a las partes un auto en el cual se plasme por escrito esta recomendación que hace el magistrado, teniendo en consideración las ventajas que aporta la mediación para las partes y en base a lo que dispone la Ley 5/2012 de mediación en asuntos civiles y mercantiles y la Directiva 2008/52/CE del Parlamento Europeo y del Consejo, de 21 de mayo de 2008, sobre ciertos aspectos de la mediación en asuntos civiles y mercantiles.

17 GARCÍA VILLALUENGA, L., VAZQUEZ DE CASTRO, E., La Mediación a debate en Europa ¿Hacia una voluntariedad mitigada? en Anuario de Mediación y solución de conflictos 2015. AA.VV., GARCÍA VILLALUENGA L., VÁZQUEZ DE CASTRO E., Directores, Madrid, Ed. Reus, 2016, pp. 21-36.

18 Consejo General de la Abogacía. Abogados de Badajoz 2014, Conclusiones /l encuentro de abogados y abogadas de violencia de genero, (en línea). Disponible en: http://www.abogacia.es/wpcontent/uploads/2015/03/CONCLUSIONES-VG-BADAJOZ.pdf.

19 Unión Europea, Comisión de las Comunidades Europeas, Libro Verde sobre las modalidades alternativas de solución de conflictos en el ámbito del Derecho Civil y Mercantil, Bruselas, Unión Europea. Comisión de las Comunidades Europeas, 2002, p. 2. Disponible en: http://www.poderjudicial.es/cgpj/es/Temas/Mediacion/Normativa-y-jurisprudencia/Normativa-europea/LibroVerde-sobre-las-modalidades-alternativas-de-solucion-de-conflictos-en-el-ambito-del-derechocivil-y-mercantil.

20 Ley 5/2012, de 6 de julio, de mediación en asuntos civiles y mercantiles. 